Internet Engineering Task Force (IETF)

R. Droms

Request for Comments: 7083

Updates: 3315

Category: Standards Track

ISSN: $2070-1721$

Modification to Default Values of SOL_MAX_RT and INF_MAX_RT

Abstract

This document updates RFC 3315 by redefining the default values for SOL_MAX_RT and INF_MAX_RT and defining options through which a DHCPV6 server can override the client's default value for SOL_MAX_RT and INF_MAX_RT with new values.

Status of This Memo

This is an Internet Standards Track document.

This document is a product of the Internet Engineering Task Force (IETF). It represents the consensus of the IETF community. It has received public review and has been approved for publication by the Internet Engineering Steering Group (IESG). Further information on Internet standards is available in section 2 of RFC 5741.

Information about the current status of this document, any errata, and how to provide feedback on it may be obtained at

http://www.rfc-editor.org/info/rfc7083.

Copyright Notice

Copyright (c) 2013 IETF Trust and the persons identified as the document authors. All rights reserved.

This document is subject to BCP 78 and the IETF Trust's Legal Provisions Relating to IETF Documents

(http://trustee.ietf.org/license-info) in effect on the date of publication of this document. Please review these documents carefully, as they describe your rights and restrictions with respect to this document. Code Components extracted from this document must include Simplified BSD License text as described in Section 4.e of the Trust Legal Provisions and are provided without warranty as described in the Simplified BSD License. 
Table of Contents

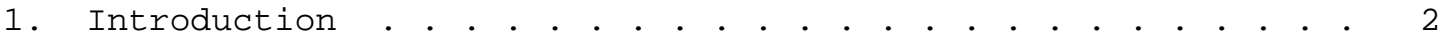

2. Requirements Language . . . . . . . . . . . . . . . . . . . 2

3. Updates to SOL_MAX_RT and INF_MAX_RT in RFC 3315 . . . . . . 2

4. SOL_MAX_RT option . . . . . . . . . . . . . . . . . . . . 3

5. INF_MAX_RT Option . . . . . . . . . . . . . . . . . . . . . . . . 4

6. Updates for SOL_MAX_RT and INF_MAX_RT Options to RFC 3315 • . 4

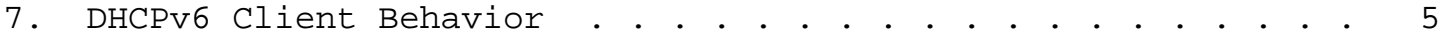

8. DHCPv6 Server Behavior . . . . . . . . . . . . . . . . . 6

9. DHCPv6 Relay Agent Behavior . . . . . . . . . . . . . . . 6

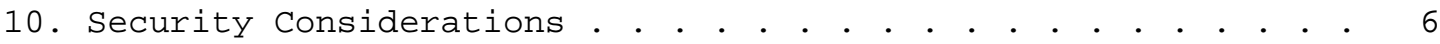

11. Acknowledgments . • • • • • • • • • • • • • • • • • • • • •

12. IANA Considerations . . . . . . . . . . . . . . . . . . . . . 7

13. References . . . . . . . . . . . . . . . . . . . . . . 7

13.1. Normative References . . . . . . . . . . . . . . . .7

13.2. Informative References . . . . . . . . . . . . . . . . 7

1. Introduction

Section 5.5 of the DHCPv6 specification [RFC3315] defines the default values of SOL_MAX_RT and INF_MAX_RT to be 120 seconds. In some circumstances, these defaults will lead to an unacceptably high volume of aggregated traffic at a DHCPv6 server.

The change to SOL_MAX_RT is in response to DHCPV6 message rates observed on a DHCPv6 server in a deployment in which many DHCPv6 clients are sending solicit messages, but the DHCPv6 server has been configured not to respond to those Solicit messages. While no explicit observations of traffic due to INF_MAX_RT have been conducted, this document updates INF_MAX_RT for consistency with SOL_MAX_RT.

\section{Requirements Language}

The key words "MUST", "MUST NOT", "REQUIRED", "SHALL", "SHALL NOT", "SHOULD", "SHOULD NOT", "RECOMMENDED", "MAY", and "OPTIONAL" in this document are to be interpreted as described in RFC 2119 [RFC2119].

3. Updates to SOL_MAX_RT and INF_MAX_RT in RFC 3315

This document changes section 5.5 of RFC 3315 as follows:

OLD :

SOL_MAX_RT 120 secs Max Solicit timeout value

NEW :

SOL_MAX_RT $\quad 3600$ secs Max Solicit timeout value 
OLD :

INF_MAX_RT 120 secs Max Information-request timeout value

NEW :

INF_MAX_RT 3600 secs Max Information-request timeout value

With this change, a DHCPv6 client that does not receive a satisfactory response will send Solicit or Information-request messages with the same initial frequency and exponential backoff as specified in Sections 17.1 .2 and 18.1 .5 of RFC 3315. However, the long-term behavior of these DHCPv 6 clients will be to send a Solicit or Information-request message every 3600 seconds rather than every 120 seconds, significantly reducing the aggregated traffic at the DHCPv6 server.

4. SOL_MAX_RT option

A DHCPv6 server sends the SOL_MAX_RT option to a client to override the default value of SOL_MAX_RT. The value of SOL_MAX_RT in the option replaces the default value defined in section 3. One use for the SOL_MAX_RT option is to set a longer value for SOL_MAX_RT, which reduces the Solicit traffic from a client that has not received a response to its solicit messages.

The format of the SOL_MAX_RT option is:

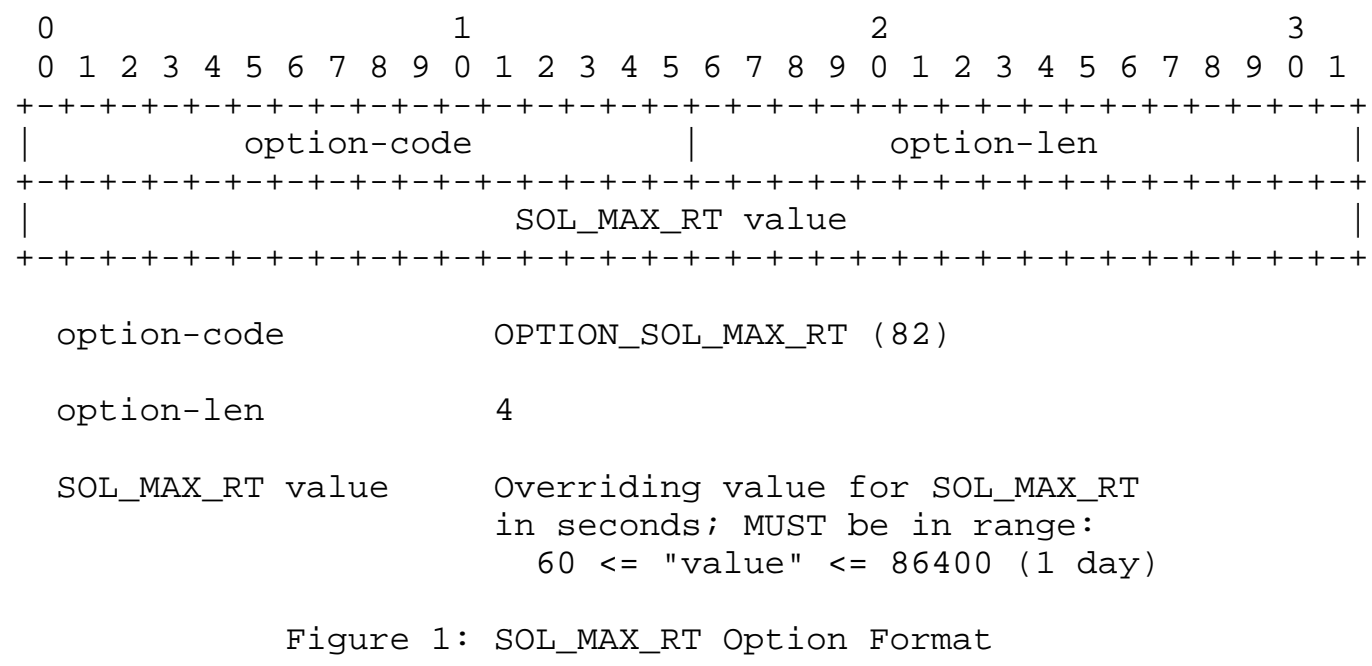

Figure 1: SOL_MAX_RT Option Format 
5. INF_MAX_RT Option

A DHCPV6 server sends the INF_MAX_RT option to a client to override the default value of INF_MAX_RT. The value of INF_MAX_RT in the option replaces the default value defined in section 3. One use for the INF_MAX_RT option is to set a longer value for INF_MAX_RT, which reduces the Information-request traffic from a client that has not received a response to its Information-request messages.

The format of the INF_MAX_RT option is:

$\begin{array}{llllllllllllllllllllllllllllllll}0 & 1 & 2 & 3 & 4 & 5 & 6 & 7 & 8 & 9 & 0 & 1 & 2 & 3 & 4 & 5 & 6 & 7 & 8 & 9 & 0 & 1 & 2 & 3 & 4 & 5 & 6 & 7 & 8 & 9 & 0\end{array}$

Figure 2: INF_MAX_RT Option Format

6. Updates for SOL_MAX_RT and INF_MAX_RT Options to RFC 3315

Update to RFC 3315, Section 17.1.3:

OLD :

The client MUST ignore any Advertise message that includes a Status Code option containing the value NoAddrsAvail, with the exception that the client MAY display the associated status message to the user.

NEW :

The client MUST ignore any Advertise message that includes a status Code option containing the value NoAddrsAvail, with the exception that the client MUST process an included SOL_MAX_RT option, MUST process an included INF_MAX_RT option, and MAY display the associated status message to the user. 
Update to RFC 3315, Section 17.2.2:

OLD :

If the server will not assign any addresses to any IAs in a subsequent Request from the client, the server MUST send an Advertise message to the client that includes only a status code option with code NoAddrsAvail and a status message for the user, a Server Identifier option with the server's DUID, and a Client Identifier option with the client's DUID.

NEW :

If the server will not assign any addresses to any IAs in a subsequent Request from the client, the server MUST send an Advertise message to the client that includes only a Status Code option with code NoAddrsAvail and a status message for the user, a Server Identifier option with the server's DUID, a Client Identifier option with the client's DUID, and (optionally) SOL_MAX_RT and/or INF_MAX_RT options.

Update to RFC 3315, Section 14 (Add text, clarifying client behavior while waiting for a response from a server):

NEW :

A client is not expected to listen for a response during the entire period between transmission of Solicit or Information-request messages.

7. DHCPv6 Client Behavior

A DHCPv6 client MUST include the SOL_MAX_RT option code in any Option Request option [RFC3315] it sends as required by $\mathrm{RFC} 3315$.

A DHCPv6 client MUST include the INF_MAX_RT option code in any Option Request option it sends as required by RFC 3315.

A DHCPv6 client MUST silently ignore any SOL_MAX_RT or INF_MAX_RT option values that are less than 60 or more than 86400 .

If a DHCPv6 client receives a message containing a SOL_MAX_RT option that has a valid value for SOL_MAX_RT, the client MUST set its internal SOL_MAX_RT parameter to the value contained in the SOL_MAX_RT option. This value of SOL_MAX_RT is then used by the retransmission mechanism defined in sections 14 and 17.1 .2 of RFC 3315 . 
If a DHCPv6 client receives a message containing an INF_MAX_RT option that has a valid value for INF_MAX_RT, the client MUST set its internal INF_MAX_RT parameter to the value contained in the INF_MAX_RT option. This value of INF_MAX_RT is then used by the retransmission mechanism defined in Sections 14 and 18.1 .5 of $\mathrm{RFC}$ 3315 .

Updated SOL_MAX_RT and INF_MAX_RT values apply only to the network interface on which the client received SOL_MAX_RT and/or INF_MAX_RT options.

8. DHCPv6 Server Behavior

Sections 17.2 .2 and 18.2 of RFC 3315 govern server operation in regard to option assignment. As a convenience to the reader, we mention here that the server will send options SOL_MAX_RT and INF_MAX_RT only if configured with specific values for them, and the client requested those options using the Option Request option.

The DHCPv6 server MAY include the SOL_MAX_RT option in any response it sends to a client that has included the SOL_MAX_RT option code in an Option Request option. The SOL_MAX_RT option is sent in the main body of the message to client, not as an encapsulated option in, e.g., an IA_NA, IA_TA [RFC3315], or IA_PD [RFC3633] option.

The DHCPv6 server MAY include the INF_MAX_RT option in any response it sends to a client that has included the INF_MAX_RT option code in an Option Request option. The INF_MAX_RT option is sent in the main body of the message to client, not as an encapsulated option in, e.g., an IA_NA, IA_TA, or IA_PD option.

9. DHCPv6 Relay Agent Behavior

There are no additional requirements for relays.

10. Security Considerations

This document introduces one security consideration beyond those described in RFC 3315. A malicious DHCPV6 server might cause a client to set its SOL_MAX_RT and INF_MAX_RT parameters to an unreasonably high value with the SOL_MAX_RT and INF_MAX_RT options, which may cause an undue delay in a client completing its DHCPv6 protocol transaction in the case no other valid response is received. Assuming the client also receives a response from a valid DHCPv6 server, large values for SOL_MAX_RT and INF_MAX_RT will not have any effect. 


\section{Acknowledgments}

Tomek Mrugalski edited the text for compliance with "Guidelines for Creating New DHCPv6 Options" [DHC-OPTION] and added important details to the Security Considerations section.

\section{IANA Considerations}

IANA has assigned one option code each for OPTION_SOL_MAX_RT (82) and OPTION_INF_MAX_RT (83) from the "DHCP Option Codes" table of the "Dynamic Host Configuration Protocol for IPv6 (DHCPv6)" registry.

13. References

13.1. Normative References

[RFC2119] Bradner, S., "Key words for use in RFCs to Indicate Requirement Levels", BCP 14, RFC 2119, March 1997.

[RFC3315] Droms, R., Bound, J., Volz, B., Lemon, T., Perkins, C., and M. Carney, "Dynamic Host Configuration Protocol for IPv6 (DHCPv6)", RFC 3315, July 2003.

13.2. Informative References

[DHC-OPTION]

Hankins, D., Mrugalski, T., Siodelski, M., Jiang, S., and S. Krishnan, "Guidelines for Creating New DHCPv6 Options", Work in Progress, September 2013.

[RFC3633] Troan, O. and R. Droms, "IPv6 Prefix Options for Dynamic Host Configuration Protocol (DHCP) version 6", RFC 3633, December 2003 .

Author's Address

Ralph Droms Cisco Systems

1414 Massachusetts Avenue Boxborough, MA 01719

USA

Phone: +1 9789361674

EMail: rdroms@cisco.com 